\title{
Almost global existence for Hamiltonian semi-linear Klein-Gordon equations with small Cauchy data on Zoll manifolds
}

\author{
D. Bambusi, J.-M. Delort, B. Grébert, J. Szeftel
}

\begin{abstract}
This paper is devoted to the proof of almost global existence results for Klein-Gordon equations on Zoll manifolds (e.g. spheres of arbitrary dimension) with Hamiltonian nonlinearities, when the Cauchy data are smooth and small. The proof relies on Birkhoff normal form methods and on the specific distribution of eigenvalues of the laplacian perturbed by a potential on Zoll manifolds.
\end{abstract}

\section{Introduction}

Let $(M, g)$ be a compact Riemannian manifold without boundary, denote by $\Delta_{g}$ its Laplace-Beltrami operator, and consider the nonlinear Klein-Gordon equation

$$
\left(\partial_{t}^{2}-\Delta_{g}+V+m^{2}\right) v=-\partial_{2} f(x, v)
$$

where $m$ is a strictly positive constant, $V$ is a smooth nonnegative potential on $M$ and $f \in C^{\infty}(M \times \mathbb{R})$ vanishes at least at order 3 in $v, \partial_{2} f$ being the derivative with respect to the second variable. In this work we prove that, for a special class of manifolds and for almost every value of $m>0$, this Hamiltonian partial differential equation admits a Birkhoff normal form at any order. The principal dynamical consequence is the almost global existence of small amplitude solutions for such a nonlinear Klein-Gordon equation.

More precisely, if $M$ is a Zoll manifold (i.e. a compact manifold whose geodesic flow is periodic, e.g. a sphere), for almost every value of $m>0$ and for any $N \in \mathbb{N}$, we prove that there is $s \gg 1$ such that, if the initial data $\left(\left.v\right|_{t=0},\left.\partial_{t} v\right|_{t=0}\right)$ are of size $\epsilon \ll 1$ in $H^{s} \times H^{s-1}$, (1.1) has a solution defined on a time interval of length $C_{N} \epsilon^{-N}$. As far as we know, this is the first result of that type when the dimension of the manifold is larger or equal to 2 . 
Let us recall some known results for the similar problem on $\mathbb{R}^{d}$, when the Cauchy data are smooth, compactly supported, of size $\epsilon \ll 1$. In this case, linear solutions decay in $L^{\infty}$ like $t^{-d / 2}$ when $t \rightarrow \infty$. This allows one to get global solutions including quasi-linear versions of (1.1), when $d \geq 2$ (see Klainerman [19] and Shatah [25] if $d \geq 3$ and Ozawa, Tsutaya and Tsutsumi [24] if $d=2$ ). When $d=1$ Moriyama, Tonegawa and Tsutsumi [21] proved that solutions exist over intervals of time of exponential length $e^{c / \epsilon^{2}}$. This result is in general optimal (see references in [12]), but global existence for small $\epsilon>0$ was proved in [12] when the nonlinearity satisfies a special condition (a "null condition" in the terminology introduced by Klainerman in the case of the wave equation in 3-space dimensions [18]).

For the problem we are studying here, since we have no dispersion on a compact manifold, we cannot hope to exploit any time decay of the solutions of the linear equation. Instead we shall use a normal form method. Remark that if in (1.1) the nonlinearity vanishes at order $p \geq 2$ at $v=0$, local existence theory gives a solution defined on an interval of length $c \epsilon^{-p+1}$. Recently, in [13], [14] Delort and Szeftel proved that the solution of the same equation exists, for almost all $m>0$, over a time interval of length $c \epsilon^{-q+1}$, where $q$ is an explicit number strictly larger than $p$ (typically $q=2 p-1$ ). Actually these papers concern more general nonlinearities than the one in (1.1), namely a suitable class of non Hamiltonian nonlinearities depending on time and space derivatives of $v$.

One of the ideas developed by Delort-Szeftel consists in reducing, by normal form procedure, (1.1) to a new system in which the nonlinearity vanishes at order $q>p$ at the origin. In [14] an explicit computation showed that the first order normal form (which leads to a nonlinearity of degree $q$ ) conserves also the $H^{s}$ norm for any large $s$, whence the result cited above.

On the other hand in [2] Bambusi and Grébert proved an abstract Birkhoff normal form theorem for Hamiltonian PDEs. Although that theorem remains valid in all dimensions, it supposes that the nonlinearity satisfies a "tame modulus" property. In [2] this property was only verified for a quite general class of $1-d$ PDEs and for a particular NLS equation on the torus $\mathbb{T}^{d}$ with arbitrary $d$. Actually in that paper, the tame modulus property was verified by the use of the property of "well localization with respect to the exponentials" established by Craig and Wayne [10], a property which has no equivalent in higher dimensions.

It turns out that in [14] Delort and Szeftel proved an estimate concerning multilinear forms defined on $M$ that implies a weaker form of the tame modulus property assumed in [2]. The present paper is the result of the combination of the arguments of [13], [14] and of [2]. 
We recall that some other partial normal form results for PDEs have been previously obtained by Kuksin and Pöschel [20], by Bourgain [5, 7] and, for perturbations of completely resonant systems, by Bambusi and Nekhoroshev [3]. For a more precise discussion we refer to the introduction of [2].

Let us conclude this introduction mentioning several open questions. The first concerns the possibility of proving almost global existence for more general nonlinearities than the Hamiltonian ones we consider here. Of course, one cannot expect to be able to do so for any nonlinearity depending on $v$ and its first order derivatives: in [11] an example is given on the circle $\mathbb{S}^{1}$ of a nonlinearity for which the solution does not exist over a time interval of length larger than the one given by local existence theory (Remark that this example holds true for any value of $m>0)$. On the other hand, Delort and Szeftel constructed in [15] almost global solutions of equations of type (1.1) on manifolds of revolution, for radial data, with a nonlinearity $f$ depending on $\left(v, \partial_{t} v\right)$ and even in $\partial_{t} v$. We thus ask the question of finding a "null condition" (in the spirit of Klainerman [18]) for semi-linear nonlinearities $f\left(v, \partial_{t} v, \nabla v\right)$, which would allow almost global existence of small $H^{s}$ solutions for almost every $m>0$.

The second question we would like to mention concerns the exceptional values of $m$ which are excluded of our result. The conservation of the Hamiltonian of equation (1.1) allows one to control the $H^{1}$-norm of small solutions. This implies global existence of small $H^{1}$ solutions in one or two space dimensions. The results we establish in the present paper show that for almost every $m>0$, the $H^{s}$-norms of these solutions remain small over long time intervals if they are so at $t=0$. What happens when $m$ is in the exceptional set? In [6] Bourgain constructed, in one space dimension and for a convenient perturbation of $-\Delta$, an example of a solution whose $H^{s}$-norm grows with time. Nothing seems to be known in larger dimensions. In particular, if $d \geq 3$, one does not even know if for all $m>0$ a solution exists almost globally, eventually without staying small in $H^{s}(s \gg 1)$.

\section{Statement of main results}

We begin, in section 2.1, by a precise exposition of our result concerning the almost globality. The Birkhoff normal form theorem for equation (1.1) that implies the almost globality result will be presented in section 2.3 , after the introduction of the Hamiltonian formalism in section 2.2. 


\subsection{Almost global solution}

Let $(M, g)$ be a compact Riemannian manifold without boundary of dimension $d \geq 1$. Denote by $\Delta_{g}$ its Laplace-Beltrami operator. Let $V$ be a smooth nonnegative potential on $M$ and $m \in(0, \infty)$. Let $f \in C^{\infty}(M \times \mathbb{R})$ be such that $f$ vanishes at least at order 3 in $v$. We consider the following Cauchy problem for the nonlinear Klein-Gordon equation

$$
\begin{aligned}
\left(\partial_{t}^{2}-\Delta_{g}+V+m^{2}\right) v & =-\partial_{2} f(x, v) \\
\left.v\right|_{t=0} & =\epsilon v_{0} \\
\left.\partial_{t} v\right|_{t=0} & =\epsilon v_{1}
\end{aligned}
$$

where $v_{0} \in H^{s}(M, \mathbb{R}), v_{1} \in H^{s-1}(M, \mathbb{R})$ are real valued given data and $\epsilon>0$. We shall prove that the above problem has almost global solutions for almost every $m$ when $\epsilon>0$ is small enough and $s$ is large enough, under the following geometric assumption on $M$ :

Definition 2.1. One says that $(M, g)$ is a Zoll manifold if and only if the geodesic flow is periodic on the cosphere bundle of $M$.

Our main dynamical result is the following:

Theorem 2.2. Let $(M, g)$ be a Zoll manifold and let $V: M \rightarrow \mathbb{R}$ be a smooth nonnegative potential. Let $r \in \mathbb{N}$ be an arbitrary integer. There is a zero measure subset $\mathcal{N}$ of $(0,+\infty)$, and for any $m \in(0,+\infty) \backslash \mathcal{N}$, there is $s_{0} \in \mathbb{N}$ such that for any $s \geq s_{0}$, for any real valued $f \in C^{\infty}(M \times \mathbb{R})$ vanishing at least at order 3 at $v=0$, there are $\epsilon_{0}>0, c>0$, such that for any pair $\left(v_{0}, v_{1}\right)$ of real valued functions belonging to the unit ball of $H^{s}(M, \mathbb{R}) \times H^{s-1}(M, \mathbb{R})$, any $\epsilon \in\left(0, \epsilon_{0}\right)$, the Cauchy problem (2.1) has a unique solution

$$
v \in C^{0}\left(\left(-T_{\epsilon}, T_{\epsilon}\right), H^{s}(M, \mathbb{R})\right) \cap C^{1}\left(\left(-T_{\epsilon}, T_{\epsilon}\right), H^{s-1}(M, \mathbb{R})\right)
$$

with $T_{\epsilon} \geq c \epsilon^{-r}$. Moreover there is $C>0$ such that, for any $t \in\left(-T_{\epsilon}, T_{\epsilon}\right)$, one has

$$
\|v(t, \cdot)\|_{H^{s}}+\left\|\partial_{t} v(t, \cdot)\right\|_{H^{s-1}} \leq C \epsilon .
$$

\section{Comments}

The above theorem provides Sobolev bounded almost global solutions for equation (2.1) with small smooth Cauchy data on a convenient class of compact manifolds. To our knowledge this is the first result of this kind on compact manifolds of dimension larger or equal to 2. In the case of one dimensional compact manifolds, similar statements have been obtained by Bourgain $[5,7]$ (with a loss on the number of derivatives of the solution with respect to those of the data), by Bambusi [4] and by Bambusi-Grébert [2]. Remark that in this case, because 
of the conservation of the Hamiltonian of the equation, one controls uniformly the $H^{1}$-norm of small solutions, which implies global existence of such solutions. The results of the preceding authors allow to control $H^{s}$-norms of these solutions for very long times. In the case of compact manifolds of revolution and for convenient radial data, Delort and Szeftel got in [15] Sobolev bounded almost global solutions (remark that this result is morally one-dimensional).

The assumption that $M$ is a Zoll manifold will be used in the proof through distribution properties of the eigenvalues of the Laplacian of $M$. Actually we shall prove theorem 2.2 for any compact manifold without boundary $(M, g)$ such that if

$$
P=\sqrt{-\Delta_{g}+V}
$$

the spectrum $\sigma(P)$ of $P$ satisfies the following condition: there are constants $\tau>0, \alpha \in \mathbb{R}, c_{0}>0, \delta>0, C_{0}>0, D \geq 0$, and a family of disjoint compact intervals $\left(K_{n}\right)_{n \geq 1}$, with $K_{1}$ at the left of $K_{2}$ and for $n \geq 2$

$$
K_{n}=\left[\frac{2 \pi}{\tau} n+\alpha-\frac{c_{0}}{n^{\delta}}, \frac{2 \pi}{\tau} n+\alpha+\frac{c_{0}}{n^{\delta}}\right]
$$

such that

$$
\begin{aligned}
& \sigma(P) \subset \bigcup_{n \geq 1} K_{n} \\
& \#\left(\sigma(P) \cap K_{n}\right) \leq C_{0} n^{D} .
\end{aligned}
$$

If $M$ is a Zoll manifold, and if $\tau>0$ is the minimal period of the geodesic flow on $M$, the results of Colin de Verdière [9] (see also Guillemin [17] and Weinstein [26]) show that the large eigenvalues of $P$ are contained inside the union of the intervals

$$
\left[\frac{2 \pi}{\tau} n+\alpha-\frac{C}{n}, \frac{2 \pi}{\tau} n+\alpha+\frac{C}{n}\right]
$$

for $n$ large enough and for some constant $C>0$. Making a translation in $n$ and $\alpha$, and changing the definition of the constants, one sees that this implies conditions $(2.4),(2.5)$ for any $\delta \in(0,1)$ (remark that the second condition in (2.5) holds true with $D=d-1$ because of Weyl law).

On the other hand conditions (2.4), (2.5) are not more general than the assumption that $M$ is a Zoll manifold, since by theorem 3.2 in Duistermaat and Guillemin [16], they imply that the geodesic flow is periodic.

\subsection{Hamiltonian formalism}

We introduce here (see e.g. [8]) the Hamiltonian formalism we shall use to solve the equation. We denote by

$$
\left\langle f_{1}, f_{2}\right\rangle
$$


the bilinear pairing between complex valued distributions and test functions on $M$. We shall use the same notation for vector valued $f_{1}, f_{2}$.

If $F$ is a $C^{\infty}$ function on an open subset $\mathcal{U}$ of the Sobolev space of real valued functions $H^{s}(M, \mathbb{R}), s \geq 0$, we define for $p \in \mathcal{U}$, the $L^{2}$ gradient $\nabla F(p)$ by

$$
\partial F(p) h=\langle\nabla F(p), h\rangle, \quad \forall h \in H^{s}(M, \mathbb{R}),
$$

$\partial F$ denoting the differential. In that way $\nabla F(p)$ is an element of $H^{-s}(M, \mathbb{R})$. When we consider real valued $C^{\infty}$ functions defined on an open subset of $H^{s}(M, \mathbb{R}) \times H^{s}(M, \mathbb{R}) \equiv H^{s}(M, \mathbb{R})^{2},(p, q) \mapsto F(p, q)$ we write

$$
\begin{aligned}
\partial F(p, q) & =\left(\partial_{p} F(p, q), \partial_{q} F(p, q)\right) \\
\nabla F(p, q) & =\left(\nabla_{p} F(p, q), \nabla_{q} F(p, q)\right) \in H^{-s}(M, \mathbb{R}) \times H^{-s}(M, \mathbb{R}) .
\end{aligned}
$$

Endow $H^{s}(M, \mathbb{R})^{2}$ with the weak symplectic structure

$$
\Omega\left((p, q),\left(p^{\prime}, q^{\prime}\right)\right):=\left\langle q, p^{\prime}\right\rangle-\left\langle q^{\prime}, p\right\rangle=\left\langle J^{-1}(p, q),\left(p^{\prime}, q^{\prime}\right)\right\rangle
$$

where $J$ is given by

$$
J=\left[\begin{array}{cc}
0 & -1 \\
\mathbf{1} & 0
\end{array}\right] .
$$

If $\mathcal{U}$ is an open subset of $H^{s}(M, \mathbb{R})^{2}$ and $F \in C^{\infty}(\mathcal{U}, \mathbb{R})$, then, for $(p, q) \in \mathcal{U}$, we define its Hamiltonian vector field by

$$
X_{F}(p, q)=J \nabla F(p, q)=\left(-\nabla_{q} F(p, q), \nabla_{p} F(p, q)\right)
$$

which is characterized by

$$
\Omega\left(X_{F},\left(h_{p}, h_{q}\right)\right)=\partial F\left(h_{p}, h_{q}\right)=\partial_{p} F h_{p}+\partial_{q} F h_{q}
$$

for any $\left(h_{p}, h_{q}\right) \in H^{s}(M, \mathbb{R})^{2}$.

A special role is played by the functions whose Hamiltonian vector field is an $H^{s}(M, \mathbb{R})^{2}$ valued function. Thus we give the following

Definition 2.3. If $\mathcal{U}$ is an open subset of $H^{s}(M, \mathbb{R})^{2}$, we denote by $C_{s}^{\infty}(\mathcal{U}, \mathbb{R})$ $\left(\right.$ resp. $\left.C_{s}^{\infty}(\mathcal{U}, \mathbb{C})\right)$ the space of real (resp. complex) valued $C^{\infty}$ functions defined on $\mathcal{U}$ such that

$$
X_{F} \in C^{\infty}\left(\mathcal{U}, H^{s}(M, \mathbb{R})^{2}\right) \quad\left(\text { or } \quad \nabla F \in C^{\infty}\left(\mathcal{U}, H^{s}(M, \mathbb{R})^{2}\right)\right),
$$

resp.

$$
X_{F} \in C^{\infty}\left(\mathcal{U}, H^{s}(M, \mathbb{R})^{2} \otimes \mathbb{C}\right) \quad\left(\text { or } \quad \nabla F \in C^{\infty}\left(\mathcal{U}, H^{s}(M, \mathbb{R})^{2} \otimes \mathbb{C}\right)\right) .
$$


We shall use complex coordinates in $H^{s}(M, \mathbb{R})^{2}$ identifying this space with $H^{s}(M, \mathbb{C})$, through $(p, q) \mapsto u=(p+\mathrm{i} q) / \sqrt{2}$. We set

$$
\begin{array}{cc}
\partial_{u}=\frac{1}{\sqrt{2}}\left(\partial_{p}-\mathrm{i} \partial_{q}\right), & \partial_{\bar{u}}=\frac{1}{\sqrt{2}}\left(\partial_{p}+\mathrm{i} \partial_{q}\right) \\
\nabla_{u}=\frac{1}{\sqrt{2}}\left(\nabla_{p}-\mathrm{i} \nabla_{q}\right), & \nabla_{\bar{u}}=\frac{1}{\sqrt{2}}\left(\nabla_{p}+\mathrm{i} \nabla_{q}\right)
\end{array}
$$

so that, if $F$ is a $C^{1}$ real valued function, we have an identification

$$
X_{F}(u, \bar{u})=\mathrm{i} \nabla_{\bar{u}} F(u, \bar{u})
$$

If $F \in C_{s}^{\infty}(\mathcal{U}, \mathbb{R})$, then clearly $X_{F} \in C^{\infty}\left(\mathcal{U}, H^{s}(M, \mathbb{C})\right)$.

For $m \in(0,+\infty)$ let us define

$$
\Lambda_{m}=\sqrt{-\Delta_{g}+V+m^{2}} .
$$

Let $s>(d-1) / 2$. We shall write equation (1.1) as a Hamiltonian system for $p=\Lambda_{m}^{-1 / 2} \partial_{t} v$ and $q=\Lambda_{m}^{1 / 2} v$ on $H^{s}(M, \mathbb{R})^{2}$. Define

$$
G_{2}(p, q)=\frac{1}{2} \int_{M}\left(\left|\Lambda_{m}^{1 / 2} p\right|^{2}+\left|\Lambda_{m}^{1 / 2} q\right|^{2}\right) d x, \quad \tilde{G}(p, q)=\int_{M} f\left(x, \Lambda_{m}^{-1 / 2} q\right) d x
$$

where $d x$ is the Riemannian volume on $M$, and set

$$
G=G_{2}+\tilde{G}
$$

Then by $(2.10)$

$$
X_{G_{2}}(p, q)=\left(-\Lambda_{m} q, \Lambda_{m} p\right), \quad X_{\tilde{G}}(p, q)=\left(-\Lambda_{m}^{-1 / 2} \partial_{2} f\left(x, \Lambda_{m}^{-1 / 2} q\right), 0\right)
$$

where $\partial_{2} f$ is the derivative with respect to the second argument. Then one has that $\tilde{G} \in C_{s}^{\infty}(\mathcal{U}, \mathbb{R})$ with $\mathcal{U}=H^{s}(M, \mathbb{R})^{2}$ (actually $X_{\tilde{G}}$ takes values in $\left.H^{s+1}(M, \mathbb{R})^{2}\right)$.

It follows also that equation (1.1) can be written as

$$
(\dot{p}, \dot{q})=X_{G}(p, q)
$$

or, using (2.16)

$$
\dot{u}=\mathrm{i} \nabla_{\bar{u}} G(u, \bar{u}) .
$$

In the rest of this section we shall give a few technical results that we shall need for the proofs of theorems 2.2, 2.6. 
Definition 2.4. Let $\mathcal{U}$ be an open subset of $H^{s}(M, \mathbb{R})^{2}$ and $F_{j} \in C_{s}^{\infty}(\mathcal{U}, \mathbb{R})$, $j=1,2$. Then their Poisson bracket is defined by

$$
\left\{F_{1}, F_{2}\right\}=\partial F_{2} \cdot X_{F_{1}}=\Omega\left(X_{F_{2}}, X_{F_{1}}\right)
$$

and one has $\left\{F_{1}, F_{2}\right\} \in C_{s}^{\infty}(\mathcal{U}, \mathbb{R})$.

One extends the definition to complex valued functions by linearity of the bracket relatively of each of its arguments.

The fact that (2.23) has a smooth vector field follows from the well known formula

$$
X_{\left\{F_{1}, F_{2}\right\}}=\left[X_{F_{1}}, X_{F_{2}}\right]=\partial X_{F_{2}} \cdot X_{F_{1}}-\partial X_{F_{1}} \cdot X_{F_{2}},
$$

with the square bracket denoting the Lie bracket of vector fields (for a proof of this formula in the case of weak symplectic manifolds see [1]). In case either $F_{1}$ or $F_{2}$ do not have a smooth vector field, one can also define their Poisson brackets by formula (2.23) but one has to check that it is a well defined function, using the fact that we may write

$$
\begin{aligned}
\left\{F_{1}, F_{2}\right\} & =-\left(\partial_{p} F_{2}\right)\left(\nabla_{q} F_{1}\right)+\left(\partial_{q} F_{2}\right)\left(\nabla_{p} F_{1}\right) \\
& =-\left\langle\nabla_{p} F_{2}, \nabla_{q} F_{1}\right\rangle+\left\langle\nabla_{q} F_{2}, \nabla_{p} F_{1}\right\rangle \\
& =\mathrm{i}\left(\partial_{u} F_{2}\right)\left(\nabla_{\bar{u}} F_{1}\right)-\mathrm{i}\left(\partial_{\bar{u}} F_{2}\right)\left(\nabla_{u} F_{1}\right) .
\end{aligned}
$$

Let us recall also the rule of transformation of vector fields and Poisson brackets under symplectomorphism. Let $\mathcal{U}$ and $\mathcal{V}$ be open subsets of $H^{s}(M, \mathbb{R})^{2}$, and $\chi: \mathcal{U} \rightarrow \mathcal{V}$ be a smooth symplectic diffeomorphism. We have by definition for any $u \in \mathcal{U}$

$$
(\partial \chi(u))^{-1}=J^{t}(\partial \chi(u)) J^{-1} .
$$

For $F \in C_{s}^{\infty}(\mathcal{V}, \mathbb{R})$ one has

$$
X_{F \circ \chi}(u)=(\partial \chi(u))^{-1} X_{F}(\chi(u))
$$

and therefore $F \circ \chi \in C_{s}^{\infty}(\mathcal{U}, \mathbb{R})$ (actually (2.27) holds in the more general context where $\nabla F$ has a domain which is left invariant by $\chi$ ). We also remark that for any $C^{1}$ real-valued function $F_{1}$ on $\mathcal{V}$ and for any $F_{2}$ in $C_{s}^{\infty}(\mathcal{V}, \mathbb{R})$ one has

$$
\left\{F_{1} \circ \chi, F_{2} \circ \chi\right\}=\left\{F_{1}, F_{2}\right\} \circ \chi .
$$

To conclude this subsection let us state as a lemma the well known formula that is the root of the Birkhoff normal form method as developed using Lie transform. 
Lemma 2.5. Let $F, G$ be two real valued functions defined on $\mathcal{U} \subset H^{s}(M, \mathbb{R})^{2}$. Assume that $F \in C_{s}^{\infty}(\mathcal{U}, \mathbb{R})$ and $G \in C^{\infty}(\mathcal{U}, \mathbb{R})$. Denote by $($ Ad $F) h=\{F, h\}$. Then $(A d F) G$ is well defined, and if we assume that for some $n \geq 1$

$$
F_{n}:=(\operatorname{AdF})^{n} G
$$

is well defined and belongs to $C_{s}^{\infty}(\mathcal{U}, \mathbb{R})$, then $F_{n+1}$ is also well defined.

Let $\mathcal{V}$ be such that $\overline{\mathcal{V}} \subset \mathcal{U}$. There exists a positive $T$ such that the flow $\mathcal{V} \ni(p, q) \mapsto \Phi^{t}(p, q) \in \mathcal{U}$ of $X_{F}$ is well defined and smooth for $|t|<T$. Moreover, for $|t|<T$ and $(p, q) \in \mathcal{V}$, one has for any $r \in \mathbb{N}$ the formula

$$
G\left(\Phi^{t}(p, q)\right)=\sum_{n=0}^{r} \frac{t^{n}}{n !} F_{n}(p, q)+\frac{1}{r !} \int_{0}^{t}(t-s)^{r} F_{r+1}\left(\Phi^{s}(p, q)\right) d s .
$$

Proof. Remark first that $(A d F) G$ is well defined by (2.25), and that under our assumptions, for $n \geq 2, F_{n}$ is well defined by definition 2.4. Since $X_{F}$ is smooth on $\mathcal{U}$ the flow $\Phi^{t}($.$) is a smooth symplectic diffeomorphism on \mathcal{V}$. For fixed $(p, q)$ put $\phi(t)=G\left(\Phi^{t}(p, q)\right)$. Formula (2.30) follows from Taylor formula since $\phi(t)$ is $C^{\infty}$. We thus have $\phi^{\prime}(t)=[(A d F) G]\left(\Phi^{t}(p, q)\right)=F_{1}\left(\Phi^{t}(p, q)\right)$. Using (2.29) one proves by induction that $\phi^{(n)}(t)=F_{n}\left(\Phi^{t}(p, q)\right)$ and the conclusion follows.

\subsection{Birkhoff Normal Form}

Using the notation of section 2.1, we define for $n \geq 1$ spectral projectors

$$
\Pi_{n}=\mathbf{1}_{K_{n}}(P) .
$$

Then, for $(p, q) \in H^{s}(M, \mathbb{R})^{2}$ we introduce the quantities

$$
J_{n}(p, q)=\frac{1}{2}\left(\left\|\Pi_{n} p\right\|_{L^{2}}^{2}+\left\|\Pi_{n} q\right\|_{L^{2}}^{2}\right) .
$$

For $(p, q) \in H^{s}(M, \mathbb{R})^{2}$ we denote

$$
\|(p, q)\|_{s}^{2}:=\|p\|_{H^{s}}^{2}+\|q\|_{H^{s}}^{2}
$$

We can now state our Birkhoff normal form result for the nonlinear KleinGordon equation on Zoll manifolds:

Theorem 2.6. Let $G$ be the Hamiltonian given by (2.18), (2.19). Then for any $r \geq 1$, there exists a zero measure subset $\mathcal{N}$ of $(0,+\infty)$, and for any $m \in(0,+\infty) \backslash \mathcal{N}$, there exists a large $s_{0}$ with the following properties: For any $s \geq s_{0}$, there exist two neighborhoods of the origin $\mathcal{U}, \mathcal{V}$, and a bijective canonical transformation $\mathcal{T}: \mathcal{V} \rightarrow \mathcal{U}$ which puts the Hamiltonian in the form

$$
G \circ \mathcal{T}=G_{2}+\mathcal{Z}+\mathcal{R}
$$


where $\mathcal{Z}$ is a real valued continuous polynomial of degree at most $r+2$ satisfying

$$
\left\{J_{n}, \mathcal{Z}\right\}=0, \quad \forall n \geq 1
$$

and $\mathcal{R} \in C_{s}^{\infty}(\mathcal{V}, \mathbb{R})$ has a zero of order $r+3$ at the origin. Precisely its vector field fulfills the estimate

$$
\left\|X_{\mathcal{R}}(p, q)\right\|_{s} \leq C_{s}\|(p, q)\|_{s}^{r+2}, \quad(p, q) \in \mathcal{V} .
$$

Finally the canonical transformation satisfies

$$
\|(p, q)-\mathcal{T}(p, q)\|_{s} \leq C_{s}\|(p, q)\|_{s}^{2}, \quad(p, q) \in \mathcal{V} .
$$

Exactly the same estimate is fulfilled on $\mathcal{U}$ by the inverse canonical transformation.

From (2.36) it follows $\mathcal{T}(0)=0$ and $\partial \mathcal{T}(0)=\mathbf{1}$.

Theorem 2.6 implies theorem 2.2 (see the proof of theorem 2.2 in section 3.3) but it says more: namely, the $J_{n}$ are almost conserved quantities for the equation (1.1). More precisely, with the notation of theorems 2.2 and 2.6, for any $n \geq 1$

$$
\left|J_{n}(p(t), q(t))-J_{n}(p(0), q(0))\right| \leq \frac{C}{n^{2 s}} \epsilon^{3} \quad \text { for }|t| \leq \epsilon^{-r}
$$

where $p(t)=\Lambda_{m}^{-1 / 2} \partial_{t} v(t)$ and $q(t)=\Lambda_{m}^{1 / 2} v(t)$ (for the proof see the end of section 3.3). Roughly speaking, the last property means that energy transfers are allowed only between modes corresponding to frequencies in the same spectral interval $K_{n}$.

\section{Proof of the main results}

In this section we prove theorem 2.6 and then deduce theorem 2.2. The proof uses a Birkhoff procedure described in subsection 3.2. Formally this procedure is very close to the classical Birkhoff scheme in finite dimension. Nevertheless, in infinite dimension, we need to define a convenient framework in order to justify the formal constructions. This framework, first introduced in [14], is presented, and adapted to our context, in the next subsection.

\subsection{Multilinear Forms}

Let us introduce some notations. If $n_{1}, \ldots, n_{k+1}$ are in $\mathbb{N}^{*}$, we denote the second and third largest elements of this family by

$$
\begin{aligned}
\max _{2}\left(n_{1}, \ldots, n_{k+1}\right) & =\max \left(\left\{n_{1}, \ldots, n_{k+1}\right\}-\left\{n_{i_{0}}\right\}\right) \\
\mu\left(n_{1}, \ldots, n_{k+1}\right) & =\max \left(\left\{n_{1}, \ldots, n_{k+1}\right\}-\left\{n_{i_{0}}, n_{i_{1}}\right\}\right)
\end{aligned}
$$


where $i_{0}$ and $i_{1}$ are the indices such that

$$
n_{i_{0}}=\max \left(n_{1}, \ldots, n_{k+1}\right), \quad n_{i_{1}}=\max _{2}\left(n_{1}, \ldots, n_{k+1}\right)
$$

and where by convention, when $k=1, \mu\left(n_{1}, n_{2}\right)=1$. We define then

$$
S\left(n_{1}, \ldots, n_{k+1}\right)=\sum_{\ell=1}^{k+1}\left[n_{\ell}-\sum_{j \neq \ell} n_{j}\right]_{+}+\mu\left(n_{1}, \ldots, n_{k+1}\right)
$$

where $[a]_{+}=\max (a, 0)$. If $n_{k}$ and $n_{k+1}$ are the largest two among $n_{1}, \ldots, n_{k+1}$, we have

$$
\begin{aligned}
& \mu\left(n_{1}, \ldots, n_{k+1}\right) \sim n_{1}+\cdots+n_{k-1}+1 \\
& S\left(n_{1}, \ldots, n_{k+1}\right) \sim\left|n_{k}-n_{k+1}\right|+n_{1}+\cdots+n_{k-1}+1 .
\end{aligned}
$$

We shall denote by $\mathcal{E}$ the algebraic direct sum of the ranges of the $\Pi_{n}$ 's defined by $(2.31)$.

Definition 3.1. Let $k \in \mathbb{N}^{*}, \nu \in[0,+\infty), N \in \mathbb{N}$.

i) We denote by $\mathcal{L}_{k+1}^{\nu, N}$ the space of $(k+1)$-linear forms $L: \mathcal{E} \times \cdots \times \mathcal{E} \rightarrow \mathbb{C}$ for which there exists $C>0$ such that for any $u_{1}, \ldots, u_{k+1} \in \mathcal{E}$, any $n_{1}, \ldots, n_{k+1}$ in $\mathbb{N}^{*}$

$$
\left|L\left(\Pi_{n_{1}} u_{1}, \ldots, \Pi_{n_{k+1}} u_{k+1}\right)\right| \leq C \frac{\mu\left(n_{1}, \ldots, n_{k+1}\right)^{\nu+N}}{S\left(n_{1}, \ldots, n_{k+1}\right)^{N}} \prod_{j=1}^{k+1}\left\|u_{j}\right\|_{L^{2}} .
$$

ii) We denote by $\mathcal{M}_{k}^{\nu, N}$ the space of $k$-linear maps $M: \mathcal{E} \times \cdots \times \mathcal{E} \rightarrow$ $L^{2}(M, \mathbb{C})$ for which there exists $C>0$ such that for any $u_{1}, \ldots, u_{k} \in \mathcal{E}$ any $n_{1}, \ldots, n_{k+1}$ in $\mathbb{N}^{*}$

$$
\left\|\Pi_{n_{k+1}} M\left(\Pi_{n_{1}} u_{1}, \ldots, \Pi_{n_{k}} u_{k}\right)\right\|_{L^{2}} \leq C \frac{\mu\left(n_{1}, \ldots, n_{k+1}\right)^{\nu+N}}{S\left(n_{1}, \ldots, n_{k+1}\right)^{N}} \prod_{j=1}^{k}\left\|u_{j}\right\|_{L^{2}} .
$$

The best constant $C$ in (3.4), (3.5) defines a norm on the above spaces. We set also $\mathcal{L}_{k+1}^{\nu,+\infty}=\bigcap_{N \in \mathbb{N}} \mathcal{L}_{k+1}^{\nu, N}$.

Consider $L \in \mathcal{L}_{k+1}^{\nu, N}$ with $N>1$ and fix an integer $j \in\{1, \ldots, k+1\}$ and elements $u_{\ell} \in \mathcal{E}$ for $\ell \in\{1, \ldots, k+1\}-\{j\}$. Then by (3.3), (3.4)

$$
\sum_{n_{j}} L\left(u_{1}, \ldots, u_{j-1}, \Pi_{n_{j}} u_{j}, u_{j+1}, \ldots, u_{k+1}\right)
$$


converges for any $u_{j} \in L^{2}(M, \mathbb{C})$, so $u_{j} \mapsto L\left(u_{1}, \ldots, u_{k+1}\right)$ extends as a continuous linear form on $L^{2}(M, \mathbb{C})$. Consequently, there is a unique element $M_{L, j}\left(u_{1}, \ldots, \widehat{u_{j}}, \ldots, u_{k+1}\right)$ of $L^{2}(M, \mathbb{C})$ with

$$
L\left(u_{1}, \ldots, u_{k+1}\right)=\left\langle u_{j}, M_{L, j}\left(u_{1}, \ldots, \widehat{u_{j}}, \ldots, u_{k+1}\right)\right\rangle
$$

for all $u_{1}, \ldots, u_{k+1} \in \mathcal{E}$. By (3.4), $M_{L, j}$ satisfies (3.5), i.e. defines an element of $\mathcal{M}_{k}^{\nu, N}$. Conversely, if we are given an element of $\mathcal{M}_{k}^{\nu, N}$, we define a multilinear form belonging to $\mathcal{L}_{k+1}^{\nu, N}$ by a formula of type (3.6).

The basic example satisfying definition 3.1 is provided by the following result proved in [14] (proposition 1.2.1).

Proposition 3.2. Let $k \in \mathbb{N}^{*}$. Denote by $d x$ any measure on $M$ with a $C^{\infty}$ density with respect to the Riemannian volume. There is $\nu \in(0,+\infty)$ such that the map

$$
\left(u_{1}, \ldots, u_{k+1}\right) \mapsto \int_{M} u_{1} \cdots u_{k+1} d x
$$

defines an element of $\mathcal{L}_{k+1}^{\nu,+\infty}$.

Remark 3.3. Up to now we did not use the spectral assumption (2.5) on the manifold $M$. Actually proposition 1.2 .1 of [14] is proved on any compact manifold without boundary, replacing in (3.4) the spectral projectors $\Pi_{n}$ defined in (2.31) by spectral projectors $\Pi_{\lambda}$ associated to arbitrary intervals of center $\lambda$ and length $O(1)$.

We now use the fundamental example given by the previous proposition to verify that the nonlinearity $\tilde{G}$ defined in (2.18) is in a good class of Hamiltonian functions. If $L$ is a $(k+1)$-linear map, and if $a \in \mathbb{N}$ satisfies $0 \leq a \leq k+1$, we set for $u, \bar{u} \in \mathcal{E}$

$$
\underline{L}^{a}(u, \bar{u})=L(u, \ldots, u, \bar{u}, \ldots, \bar{u})
$$

where in the right hand side one has $a$ times $u$ and $(k+1-a)$-times $\bar{u}$. We then define the following class of Hamiltonian functions:

Definition 3.4. For $k \in \mathbb{N}$ and $s, \nu \in \mathbb{R}$ with $s>\nu+\frac{3}{2}$, we define $\mathcal{H}_{k+1}^{s}(\nu)$ as the space of all real valued smooth functions defined on $H^{s}(M, \mathbb{C}),(u, \bar{u}) \rightarrow Q(u, \bar{u})$, such that there are for $\ell=0, \ldots, k+1$ multilinear forms $L_{\ell} \in \mathcal{L}_{k+1}^{\nu,+\infty}$ with

$$
Q(u, \bar{u})=\sum_{\ell=0}^{k+1} \underline{L}_{\ell}^{\ell}(u, \bar{u}) .
$$

This definition is obtain by adapting to our context the usual definition of polynomial used for example in the theory of analytic functions on Banach spaces (see for example [22] or [23]).

As a consequence of proposition 3.2 one gets: 
Lemma 3.5. Let $P$ be the Taylor's polynomial of $\tilde{G}$ at degree $k$. Then there exists $\nu \in(0,+\infty)$ such that $P$ can be decomposed as

$$
P=\sum_{j=3}^{k} P_{j}
$$

where $P_{j} \in \mathcal{H}_{j}^{s}(\nu)$.

Let us recall the main properties for $\mathcal{M}_{k}^{\nu, N}$ established in proposition 2.1.3 and theorem 2.1.4 of [14].

Proposition 3.6. $\quad$ i) Let $\nu \in[0,+\infty), s \in \mathbb{R}, s>\nu+3 / 2, N \in \mathbb{N}, N>$ $s+1$. Then, any element $M \in \mathcal{M}_{k}^{\nu, N}$ extends as a bounded operator from $H^{s}(M, \mathbb{C})^{k}$ to $H^{s}(M, \mathbb{C})$. Moreover, for any $s_{0} \in(\nu+3 / 2, s]$, there is $C>0$ such that for any $u_{\ell} \in H^{s}(M, \mathbb{C}), \ell \in\{1, \ldots, k\}$

$$
\left\|M\left(u_{1}, \ldots, u_{k}\right)\right\|_{H^{s}} \leq C\|M\|_{\mathcal{M}_{k}^{\nu, N}}\left(\sum_{1 \leq \ell \leq k}\left\|u_{\ell}\right\|_{H^{s}} \prod_{\ell^{\prime} \neq \ell}\left\|u_{\ell^{\prime}}\right\|_{H^{s_{0}}}\right) .
$$

ii) Let $k_{1}, k_{2} \in \mathbb{N}^{*}, \nu_{1}, \nu_{2} \in[0,+\infty), 1 \leq \ell \leq k_{2}$. For $M_{1} \in \mathcal{M}_{k_{1}}^{\nu_{1}, N}, M_{2} \in$ $\mathcal{M}_{k_{2}}^{\nu_{2}, N}$ with $N>1+\max \left(\nu_{1}, \nu_{2}\right)$, define a $\left(k_{1}+k_{2}-1\right)$-linear operator on $\mathcal{E}^{k_{1}+k_{2}-1}$

$$
\left(u_{1}, \ldots, u_{k_{1}+k_{2}-1}\right) \rightarrow M\left(u_{1}, \ldots, u_{k_{1}+k_{2}-1}\right)
$$

by

$$
\begin{aligned}
& M\left(u_{1}, \ldots, u_{k_{1}+k_{2}-1}\right)= \\
& \quad M_{2}\left(u_{1}, \ldots, u_{\ell-1}, M_{1}\left(u_{\ell}, \ldots, u_{\ell+k_{1}-1}\right), u_{\ell+k_{1}}, \ldots, u_{k_{1}+k_{2}-1}\right) .
\end{aligned}
$$

Then $M$ belongs to $\mathcal{M}_{k_{1}+k_{2}-1}^{\nu_{1}+\nu_{2}+1, N-\max \left(\nu_{1}, \nu_{2}\right)-1}$ and the map $\left(M_{1}, M_{2}\right) \mapsto M$ is bounded from $\mathcal{M}_{k_{1}}^{\nu_{1}, N} \times \mathcal{M}_{k_{2}}^{\nu_{2}, N}$ to the preceding space.

Using the duality formula (3.6), proposition 3.6 immediately implies the corresponding properties for the multilinear forms of $\mathcal{L}_{k+1}^{\nu, N}$.

Proposition 3.7. i) Let $\nu \in[0,+\infty), s \in \mathbb{R}, s>\nu+3 / 2, N \in \mathbb{N}$, $N>s+1$. Then for any $j \in\{1, \ldots, k+1\}$, any multilinear form $L \in$ $\mathcal{L}_{k+1}^{\nu, N}$ extends as a continuous multilinear form $\left(u_{1}, \ldots, u_{j}, \ldots, u_{k+1}\right) \mapsto$ $L\left(u_{1}, \ldots, u_{j}, \ldots, u_{k+1}\right)$ on

$H^{s}(M, \mathbb{C}) \times \cdots \times H^{s}(M, \mathbb{C}) \times H^{-s}(M, \mathbb{C}) \times H^{s}(M, \mathbb{C}) \times \cdots \times H^{s}(M, \mathbb{C})$. 
Moreover for any $s_{0} \in(\nu+3 / 2, s]$, there is $C>0$ such that for any $u_{\ell} \in H^{s}(M, \mathbb{C}), \ell \in\{1, \ldots, k+1\}-\{j\}$, any $u_{j} \in H^{-s}(M, \mathbb{C})$

$$
\left|L\left(u_{1}, \ldots, u_{k+1}\right)\right| \leq C\|L\|_{\mathcal{L}_{k+1}^{\nu, N}}\left\|u_{j}\right\|_{H^{-s}}\left(\sum_{\substack{1 \leq \ell \leq k+1 \\ \ell \neq j}}\left\|u_{\ell}\right\|_{H^{s}} \prod_{\substack{\ell^{\prime} \neq \ell \\ \ell^{\prime} \neq j}}\left\|u_{\ell^{\prime}}\right\|_{H^{s}}\right) .
$$

ii) Let $k_{1}, k_{2} \in \mathbb{N}^{*}, \nu_{1}, \nu_{2} \in[0,+\infty), 1 \leq \ell \leq k_{2}+1$. For $M \in \mathcal{M}_{k_{1}}^{\nu_{1}, N}$, $L \in \mathcal{L}_{k_{2}+1}^{\nu_{2}, N}$ with $N>1+\max \left(\nu_{1}, \nu_{2}\right)$ define a $\left(k_{1}+k_{2}\right)$-linear form on $\mathcal{E}^{k_{1}+k_{2}}$

$$
\left(u_{1}, \ldots, u_{k_{1}+k_{2}}\right) \rightarrow \tilde{L}\left(u_{1}, \ldots, u_{k_{1}+k_{2}}\right)
$$

by

$$
\tilde{L}\left(u_{1}, \ldots, u_{k_{1}+k_{2}}\right)=L\left(u_{1}, \ldots, u_{\ell-1}, M\left(u_{\ell}, \ldots, u_{\ell+k_{1}-1}\right), u_{\ell+k_{1}}, \ldots, u_{k_{1}+k_{2}}\right) .
$$

Then $\tilde{L} \in \mathcal{L}_{k_{1}+k_{2}}^{\nu_{1}+\nu_{2}+1, N-\max \left(\nu_{1}, \nu_{2}\right)-1}$ and the map $(M, L) \mapsto \tilde{L}$ is bounded from $\mathcal{M}_{k_{1}}^{\nu_{1}, N} \times \mathcal{L}_{k_{2}+1}^{\nu_{2}, N}$ to the preceding space.

We shall denote, for any $N, \nu$ by

$$
\Sigma: \mathcal{L}_{k+1}^{\nu, N} \rightarrow \mathcal{M}_{k}^{\nu, N}
$$

the map given, using notation (3.6), by $\Sigma(L)=M_{L, k+1}$. This is an isomorphism.

In order to apply a Birkhoff procedure, it is necessary to verify that our framework is stable by Poisson brackets.

Proposition 3.8. Let $k_{1}, k_{2} \in \mathbb{N}^{*}, \nu_{1}, \nu_{2} \in[0,+\infty), N>\frac{5}{2}+\max \left(\nu_{1}, \nu_{2}\right)$. Let $L_{1} \in \mathcal{L}_{k_{1}+1}^{\nu_{1}, N}, L_{2} \in \mathcal{L}_{k_{2}+1}^{\nu_{2}, N}, \ell_{1} \in\left\{0, \ldots, k_{1}+1\right\}, \ell_{2} \in\left\{0, \ldots, k_{2}+1\right\}$. Then $\left\{\underline{L}_{1}^{\ell_{1}}, \underline{L}_{2}^{\ell_{2}}\right\}$ may be written

$$
\left\{\underline{L}_{1}^{\ell_{1}}, \underline{L}_{2}^{\ell_{2}}\right\}(u, \bar{u})=\underline{L}_{3}^{\ell_{1}+\ell_{2}-1}(u, \bar{u})
$$

for a multilinear form $L_{3} \in \mathcal{L}_{k_{1}+k_{2}}^{\nu_{1}+\nu_{2}+1, N-\max \left(\nu_{1}, \nu_{2}\right)-1}$.

Proof. We can choose $s$ with $N-1>s>\frac{3}{2}+\max \left(\nu_{1}, \nu_{2}\right)$. By i) of proposition 3.7, $\underline{L}_{i}^{\ell_{i}}(u, \bar{u}) i=1,2$ is then a smooth function on $H^{s}(M, \mathbb{C})$. Using (3.6) we may write for any $h \in \mathcal{E}, i=1,2$

$$
\partial_{u} \underline{L}_{i}^{\ell_{i}} \cdot h=\sum_{j=1}^{\ell_{i}} L_{i}(u, \ldots, h, \ldots, u, \bar{u}, \ldots, \bar{u})=\sum_{j=1}^{\ell_{i}}\left\langle h, \underline{M}_{L_{i}, j}^{\ell_{i}-1}(u, \bar{u})\right\rangle
$$


where in the first sum $h$ stands at the $j$-th place. We have a similar formula for $\partial_{\bar{u}} \underline{L}_{i}^{\ell_{i}} . h$. In other words, we may write

$$
\begin{aligned}
& \nabla_{u} \underline{L}_{i}^{\ell_{i}}(u, \bar{u})=\sum_{j=1}^{\ell_{i}} \underline{M}_{L_{i}, j}^{\ell_{i}-1}(u, \bar{u}) \\
& \nabla_{\bar{u}} \underline{L}_{i}^{\ell_{i}}(u, \bar{u})=\sum_{j=\ell_{i}+1}^{k_{i}+1} \underline{M}_{L_{i}, j}^{\ell_{i}}(u, \bar{u}) .
\end{aligned}
$$

By i) of proposition 3.6 these quantities are smooth functions of $u$ with values in $H^{s}(M, \mathbb{C})$, i.e. $\underline{L}_{i}^{\ell_{i}} \in C_{s}^{\infty}\left(H^{s}(M, \mathbb{C}), \mathbb{C}\right)$. We may thus apply definition 2.4 and (2.25) to write

$$
\begin{aligned}
\left\{\underline{L}_{1}^{\ell_{1}}, \underline{L}_{2}^{\ell_{2}}\right\}(u, \bar{u})=\mathrm{i} & {\left[\sum_{j_{2}=1}^{\ell_{2}} \sum_{j_{1}=\ell_{1}+1}^{k_{1}+1} L_{2}\left(u, \ldots, \underline{M}_{L_{1}, j_{1}}^{\ell_{1}}(u, \bar{u}), \ldots, u, \bar{u}, \ldots, \bar{u}\right)\right.} \\
& \left.-\sum_{j_{2}=\ell_{2}+1}^{k_{2}+1} \sum_{j_{1}=1}^{\ell_{1}} L_{2}\left(u, \ldots, u, \bar{u}, \ldots, \underline{M}_{L_{1}, j_{1}}^{\ell_{1}-1}(u, \bar{u}), \ldots, \bar{u}\right)\right]
\end{aligned}
$$

where the $M$-term in the argument of $L_{2}$ stays at the $j_{2}$-th place. Since $M_{L_{1}, j_{1}}$ belongs to $\mathcal{M}_{k_{1}}^{\nu_{1}, N}$ we just have to apply (ii) of proposition 3.7 to write this last expression in terms of a new multilinear form $L_{3}$.

In order to prove our main theorem we have to decompose the multilinear forms of $\mathcal{L}_{k+1}^{\nu, N}$ in the sum of a resonant and of a non-resonant part.

Definition 3.9. (Non-resonant multilinear form) Fix $k \in \mathbb{N}$ and let $1 \leq \ell \leq$ $k+1$ be a fixed integer.

- If $2 \ell \neq k+1$ we set $\widetilde{\mathcal{L}}_{k+1, \ell}^{\nu, N}=\mathcal{L}_{k+1}^{\nu, N}, \widetilde{\mathcal{M}}_{k, \ell}^{\nu, N}=\mathcal{M}_{k}^{\nu, N}$.

- If $2 \ell=k+1$ we define $\widetilde{\mathcal{L}}_{k+1, \ell}^{\nu, N}$ (resp. $\widetilde{\mathcal{M}}_{k, \ell}^{\nu, N}$ ) as the subspace of those $L \in \mathcal{L}_{k+1}^{\nu, N}$ (resp. $M \in \mathcal{M}_{k}^{\nu, N}$ ) such that respectively

$$
L\left(\Pi_{n_{1}} u_{1}, \ldots, \Pi_{n_{k+1}} u_{k+1}\right) \equiv 0, \quad \Pi_{n_{k+1}} M\left(\Pi_{n_{1}} u_{1}, \ldots, \Pi_{n_{k}} u_{k}\right) \equiv 0
$$

for any $u_{1}, \ldots, u_{k+1} \in \mathcal{E}$ and any $\left(n_{1}, \ldots, n_{k+1}\right) \in\left(\mathbb{N}^{*}\right)^{k+1}$ such that

$$
\left\{n_{1}, \ldots, n_{\ell}\right\}=\left\{n_{\ell+1}, \ldots, n_{k+1}\right\} .
$$

Remark that the map $\Sigma$ given by (3.13) induces an isomorphism between $\widetilde{\mathcal{L}}_{k+1, \ell}^{\nu, N}$ and $\widetilde{\mathcal{M}}_{k, \ell}^{\nu, N}$. 
Definition 3.10. (Resonant multilinear form) Fix $k \in \mathbb{N}$ and let $1 \leq \ell \leq k+1$. We define the space of $\ell$-resonant multilinear forms $\widehat{\mathcal{L}}_{k+1, \ell}^{\nu, N}$ as the subspace of those $L \in \mathcal{L}_{k+1}^{\nu, N}$ verifying

$$
L\left(\Pi_{n_{1}} u_{1}, \ldots, \Pi_{n_{k+1}} u_{k+1}\right) \equiv 0,
$$

for any $u_{1}, \ldots, u_{k+1} \in \mathcal{E}$ and any $\left(n_{1}, \ldots, n_{k+1}\right) \in\left(\mathbb{N}^{*}\right)^{k+1}$ such that

$$
\left\{n_{1}, \ldots, n_{\ell}\right\} \neq\left\{n_{\ell+1}, \ldots, n_{k+1}\right\} .
$$

Remark that $\widehat{\mathcal{L}}_{k+1, \ell}^{\nu, N}=0$ if $k$ is even or $k$ is odd and $\ell \neq \frac{k+1}{2}$. If $k$ is odd and $\ell=\frac{k+1}{2}$, one gets a direct sum decomposition

$$
\mathcal{L}_{k+1}^{\nu, N}=\widehat{\mathcal{L}}_{k+1, \ell}^{\nu, N} \oplus \tilde{\mathcal{L}}_{k+1, \ell}^{\nu, N}
$$

The main feature of the above definitions is captured by the following proposition:

Proposition 3.11. Assume that $L \in \mathcal{L}_{k+1}^{\nu, N}$ is $\ell$ resonant. Then for any $a \in \mathbb{N}$, $a \geq 1$ one has

$$
\left\{\underline{L}^{\ell}, J_{a}\right\} \equiv 0 \text {. }
$$

Proof. Remark first that one has $2 \ell=k+1$ and that $J_{a}(u, \bar{u})=\left\langle\Pi_{a} u, \Pi_{a} \bar{u}\right\rangle$, from which, using (3.16), one gets

$$
\left\{\underline{L}^{\ell}, J_{a}\right\}=-\mathrm{i} \underline{\tilde{L}}^{\ell}
$$

with

$$
\begin{aligned}
& \tilde{L}\left(u_{1}, \ldots, u_{k+1}\right)= \\
& {\left[\sum_{j=1}^{\ell} L\left(u_{1}, \ldots, \Pi_{a} u_{j}, \ldots, u_{k+1}\right)-\sum_{j=\ell+1}^{k+1} L\left(u_{1}, \ldots, \Pi_{a} u_{j}, \ldots, u_{k+1}\right)\right] .}
\end{aligned}
$$

Then the above expression is equal to

$$
\begin{aligned}
\sum_{n_{1}, \ldots, n_{k+1}}\left[\sum_{j=1}^{\ell} L\left(\Pi_{n_{1}} u_{1}, \ldots, \Pi_{a} \Pi_{n_{j}} u_{j}, \ldots, \Pi_{n_{k+1}} u_{k+1}\right)\right. & \\
& \left.-\sum_{j=\ell+1}^{2 \ell} L\left(\Pi_{n_{1}} u_{1}, \ldots, \Pi_{a} \Pi_{n_{j}} u_{j}, \ldots, \Pi_{n_{k+1}} u_{k+1}\right)\right] \\
= & \sum_{n_{1}, \ldots, n_{k+1}}\left[\sum_{j=1}^{\ell} \delta_{n_{j}, a}-\sum_{j=\ell+1}^{2 \ell} \delta_{n_{j}, a}\right] L\left(\Pi_{n_{1}} u_{1}, \ldots, \Pi_{n_{2 \ell}} u_{2 \ell}\right) .
\end{aligned}
$$


Since for an $\ell$ resonant form

$$
\left\{n_{1}, \ldots, n_{\ell}\right\}=\left\{n_{\ell+1}, \ldots, n_{2 \ell}\right\},
$$

the quantity $\sum_{j=1}^{\ell} \delta_{n_{j}, a}-\sum_{j=\ell+1}^{2 \ell} \delta_{n_{j}, a}$ always vanishes.

Definition 3.12. For given integers $\ell, k$ satisfying $1 \leq \ell \leq k+1$, we define an operator $\psi_{\ell}$ acting on $\mathcal{L}_{k+1}^{\nu, N}$ by

$$
\begin{aligned}
& \psi_{\ell}(L)\left(u_{1}, \ldots, u_{k+1}\right) \\
& =\left[\sum_{j=1}^{\ell} L\left(u_{1}, \ldots, \Lambda_{m} u_{j}, \ldots, u_{k+1}\right)-\sum_{j=\ell+1}^{k+1} L\left(u_{1}, \ldots, \Lambda_{m} u_{j}, \ldots, u_{k+1}\right)\right] .
\end{aligned}
$$

Remark that writing $G_{2}(u, \bar{u})=\left\langle\Lambda_{m} u, \bar{u}\right\rangle$, and using (2.25) one gets

$$
\left\{\underline{L}^{\ell}, G_{2}\right\}(u, \bar{u})=-\mathrm{i} \psi_{\ell}(L)(u, \ldots, u, \bar{u}, \ldots, \bar{u})
$$

where in the right hand side one has $\ell$ times $u$ and $k+1-\ell$ times $\bar{u}$.

Proposition 3.13. There is a zero measure subset $\mathcal{N}$ of $(0,+\infty)$ such that for any $k \in \mathbb{N}^{*}$, any $m \in(0,+\infty)-\mathcal{N}$, any $0 \leq \ell \leq k+1$, there is $a \bar{\nu} \in \mathbb{R}_{+}$, and for any $(\nu, N) \in \mathbb{R}_{+} \times \mathbb{N}, N>2$, there is an operator

$$
\psi_{\ell}^{-1}: \widetilde{\mathcal{L}}_{k+1, \ell}^{\nu, N} \rightarrow \widetilde{\mathcal{L}}_{k+1, \ell}^{\nu+\bar{\nu}, N}
$$

such that for any $L \in \widetilde{\mathcal{L}}_{k+1, \ell}^{\nu, N}, \psi_{\ell}\left(\psi_{\ell}^{-1}(L)\right)=L$. Moreover there exists $C>0$ such that

$$
\left\|\psi_{\ell}^{-1}(L)\right\|_{\mathcal{L}_{k+1}^{\nu+\bar{\nu}, N}} \leq C\|L\|_{\mathcal{L}_{k+1}^{\nu, N}}
$$

Proof. We reduce the proof to proposition 2.2.4 of [14]. Let $\rho:\{1, \ldots, k+1\} \rightarrow$ $\{-1,1\}$ be the map given by $\rho(j)=1$ if $j=1, \ldots, \ell$ and $\rho(j)=-1$ if $j=\ell+$ $1, \ldots, k+1$, and for $M \in \mathcal{M}_{k}^{\nu, N}$ define

$$
\begin{aligned}
& \tilde{\psi}_{\ell}(M)\left(u_{1}, \ldots, u_{k}\right)= \\
& \sum_{j=1}^{k} \rho(j) M\left(u_{1}, \ldots, \Lambda_{m} u_{j}, \ldots, u_{k}\right)+\rho(k+1) \Lambda_{m} M\left(u_{1}, \ldots, u_{k}\right) .
\end{aligned}
$$

One has, if $\Sigma$ is the map defined in (3.13),

$$
\Sigma^{-1} \circ \tilde{\psi}_{\ell}(M)=\psi_{\ell} \circ \Sigma^{-1}(M)
$$

for any $M \in \mathcal{M}_{k}^{\nu, N}$ such that $\tilde{\psi}_{\ell}(M)$ belongs to $\mathcal{M}_{k}^{\nu^{\prime}, N}$ for some $\nu^{\prime} \geq 0$. By proposition 2.2 .4 in [14], there are $\bar{\nu} \in \mathbb{R}_{+}$and an operator $\tilde{\psi}_{\ell}^{-1}: \widetilde{\mathcal{M}}_{k, \ell}^{\nu, N} \rightarrow$ 
$\widetilde{\mathcal{M}}_{k, \ell}^{\nu+\bar{\nu}, N}$ such that for any $M \in \widetilde{\mathcal{M}}_{k, \ell}^{\nu, N}, \tilde{\psi}_{\ell}\left(\tilde{\psi}_{\ell}^{-1}(M)\right)=M$ and such that the equivalent for $M$ of the estimate (3.23) holds true. We just set $\psi_{\ell}^{-1}=\Sigma^{-1}$ 。 $\tilde{\psi}_{\ell}^{-1} \circ \Sigma$, and the conclusion follows from equation (3.25).

The construction of the operator $\tilde{\psi}_{\ell}^{-1}$ in [14] relies in an essential way on the spectral assumption (2.4) and (2.5), i.e. on the fact that $M$ is a Zoll manifold. For the reader's convenience, we give a direct proof of proposition 3.13 in the case where $M=\mathbb{S}^{d}$ and $V=0$. In this case, the eigenvalues $\lambda_{n}$ of $P$ and $\omega_{n}$ of $\Lambda_{m}$ are respectively given by

$$
\lambda_{n}=\sqrt{n(n+d-1)}, \quad \omega_{n}=\sqrt{\lambda_{n}^{2}+m^{2}},
$$

and moreover $P \Pi_{n}=\lambda_{n} \Pi_{n}, \Lambda_{m} \Pi_{n}=\omega_{n} \Pi_{n}$. Thus, from equation (3.20) one has

$$
\begin{aligned}
& \psi_{\ell}(L)\left(u_{1}, \ldots, u_{k+1}\right) \\
& =\sum_{n_{1}, \ldots, n_{k+1}}\left(\omega_{n_{1}}+\cdots+\omega_{n_{\ell}}-\omega_{n_{\ell+1}}-\cdots-\omega_{n_{k+1}}\right) L\left(\Pi_{n_{1}} u_{1}, \ldots, \Pi_{n_{k+1}} u_{k+1}\right) .
\end{aligned}
$$

Remark also that, if $L \in \widetilde{\mathcal{L}}_{k+1, \ell}^{\nu, N}$, then the sum is restricted to those $\left(n_{1}, \ldots, n_{k+1}\right)$ such that

$$
\left\{n_{1}, \ldots, n_{\ell}\right\} \neq\left\{n_{\ell+1}, \ldots, n_{k+1}\right\} .
$$

The following proposition was proved in [13] (see Proposition 4.8) and is also a minor variant of theorem 3.12 of [2].

Proposition 3.14. There is a zero measure subset $\mathcal{N}$ of $(0,+\infty)$ such that for any $m \in(0,+\infty)-\mathcal{N}$ and any $k \in \mathbb{N}^{*}$, there are $c>0$ and $\bar{\nu} \in \mathbb{R}_{+}$such that for any $0 \leq \ell \leq k+1$, one has

$$
\left|\omega_{n_{1}}+\cdots+\omega_{n_{\ell}}-\omega_{n_{\ell+1}}-\cdots-\omega_{n_{k+1}}\right| \geq c \mu\left(n_{1}, \ldots, n_{k+1}\right)^{-\bar{\nu}}
$$

for any choice of $\left(n_{1}, \ldots, n_{k+1}\right)$ such that

$$
\left\{n_{1}, \ldots, n_{\ell}\right\} \neq\left\{n_{\ell+1}, \ldots, n_{k+1}\right\}
$$

It is now immediate to obtain the

Proof of Proposition 3.13 in the case $M=\mathbb{S}^{d}, V \equiv 0$. Given $L \in \widetilde{\mathcal{L}}_{k+1, \ell}^{\nu, N}$ define

$$
\tilde{L}\left(u_{1}, \ldots, u_{k+1}\right)=\sum_{n_{1}, \ldots, n_{k+1}} \frac{L\left(\Pi_{n_{1}} u_{1}, \ldots, \Pi_{n_{k+1}} u_{k+1}\right)}{\left(\omega_{n_{1}}+\cdots+\omega_{n_{\ell}}-\omega_{n_{\ell+1}}-\cdots-\omega_{n_{k+1}}\right)} .
$$


Then by (3.28) one has $\tilde{L} \in \widetilde{\mathcal{L}}_{k+1, \ell}^{\nu+\bar{\nu}, N}$, and by $(3.29) \psi_{\ell}(\tilde{L})=L$; finally also the estimate (3.23) immediately follows. On a general Zoll manifold, the construction of the map $L \rightarrow \tilde{L}$ is made in [13] through an approximation argument and a suitable use of Neumann series.

Finally we end this subsection with two lemmas that will be useful to verify that certain Hamiltonian functions are real valued.

Lemma 3.15. Assume $m \in(0,+\infty)-\mathcal{N}$ and let $L \in \widetilde{\mathcal{L}}_{k+1, \ell}^{\nu, N}$.

i) Assume that for any $u \in \mathcal{E}, \psi_{\ell}(L)(u, \ldots, u, \bar{u}, \ldots, \bar{u})=0$ (where one has $\ell$ times $u$ and $k+1-\ell$ times $\bar{u})$. Then $\underline{L}^{\ell}(u, \bar{u})=0$.

ii) Assume $\left\{\operatorname{Im} \underline{L}^{\ell}, G_{2}\right\} \equiv 0$. Then $\operatorname{Im} \underline{L}^{\ell}(u, \bar{u}) \equiv 0$.

Proof. i) Let $\mathfrak{S}_{\ell, k}$ be the product of the group of permutations of $\{1, \ldots, \ell\}$ by the group of permutations of $\{\ell+1, \ldots, k+1\}$. For $\left(\sigma, \sigma^{\prime}\right) \in \mathfrak{S}_{\ell, k}$ define

$$
\left(\left(\sigma, \sigma^{\prime}\right) \cdot L\right)\left(u_{1}, \ldots, u_{k+1}\right)=L\left(u_{\sigma(1)}, \ldots, u_{\sigma(\ell)}, u_{\sigma^{\prime}(\ell+1)}, \ldots, u_{\sigma^{\prime}(k+1)}\right) .
$$

Replacing $L$ by

$$
\frac{1}{\ell !(k+1-\ell) !} \sum_{a \in \mathfrak{S}_{\ell, k}}(a \cdot L)\left(u_{1}, \ldots, u_{k+1}\right)
$$

does no affect the hypotheses nor the conclusion (since $\psi_{\ell}$ commutes to the $\mathfrak{S}_{\ell, k}$-action), so we can assume that $L-$ and thus $\psi_{\ell}(L)-$ is $\mathfrak{S}_{\ell, k}$-invariant. Write the assumption $\psi_{\ell}(L)(u, \ldots, u, \bar{u}, \ldots, \bar{u})=0$ with

$$
u=u_{1}+\cdots+u_{\ell}+\overline{u_{\ell+1}}+\cdots+\overline{u_{k+1}}
$$

for arbitrary $u_{j}$ 's belonging to $\mathcal{E}$. If one expands this expression by multilinearity, sorts the different contributions according to their homogeneity degree in $u_{j}, \bar{u}_{j}$, and uses the $\mathfrak{S}_{\ell, k}$-invariance, one gets

$$
\psi_{\ell}(L)\left(u_{1}, \ldots, u_{k+1}\right)=0
$$

for any $u_{1}, \ldots, u_{k+1}$ in $\mathcal{E}$. Take a family of positive integers $\left(n_{1}, \ldots, n_{k+1}\right)$ such that $\left\{n_{1}, \ldots, n_{\ell}\right\} \neq\left\{n_{\ell+1}, \ldots, n_{k+1}\right\}$ if $2 \ell=k+1$. We apply (3.30) taking for all $u_{j}$ an eigenfunction associated to an eigenvalue $\lambda_{n_{j}} \in K_{n_{j}} j=1, \ldots, k+1$ so that $\Lambda_{m} u_{j}=\omega_{n_{j}} u_{j}, \omega_{n_{j}}=\sqrt{m^{2}+\lambda_{n_{j}}^{2}}$. By (3.20) we obtain

$$
\left(\sum_{j=1}^{\ell} \omega_{n_{j}}-\sum_{\ell+1}^{k+1} \omega_{n_{j}}\right) L\left(u_{1}, \ldots, u_{k+1}\right)=0 .
$$


By proposition 2.2.1 and formula (2.2.3) of [14] (see also proposition 3.14 of the present paper in the case of the sphere), the first factor is nonzero for $m \in(0,+\infty)-\mathcal{N}$, so $L\left(u_{1}, \ldots, u_{k+1}\right)=0$ for any family $\left(u_{1}, \ldots, u_{k+1}\right)$ of the preceding form. The definition of $\widetilde{\mathcal{L}}_{k+1, \ell}^{\nu, N}$ implies that $\underline{L}^{\ell}(u, \bar{u})=0$.

ii) We may write when $\ell \neq \frac{k+1}{2} \operatorname{Im} \underline{L}^{\ell}(u, \bar{u})=\underline{\Gamma}_{1}^{\ell}(u, \bar{u})+\underline{\Gamma}_{2}^{k+1-\ell}(u, \bar{u})$ for $\Gamma_{1} \in \widetilde{\mathcal{L}}_{k+1, \ell}^{\nu, N}, \Gamma_{2} \in \tilde{\mathcal{L}}_{k+1, k+1-\ell}^{\nu, N}$. By homogeneity, $\left\{G_{2}, \underline{\Gamma}_{1}^{\ell}+\underline{\Gamma}_{2}^{k+1-\ell}\right\} \equiv 0$ implies that $\left\{G_{2}, \underline{\Gamma}_{1}^{\ell}\right\}=\left\{G_{2}, \underline{\Gamma}_{2}^{k+1-\ell}\right\} \equiv 0$, whence $\underline{\Gamma}_{1}^{\ell}=\underline{\Gamma}_{2}^{k+1-\ell}=0$ by (3.21) and assertion i). If $\ell=\frac{k+1}{2}$, we have $\operatorname{Im} \underline{L}^{\ell}(u, \bar{u})=\underline{\Gamma}^{\ell}(u, \bar{u})$ for a $\Gamma \in \widetilde{\mathcal{L}}_{k+1}^{\nu, N}$, and the result follows again from $\mathrm{i}$ ).

Lemma 3.16. Assume $m \in(0,+\infty)-\mathcal{N}$ and $k$ odd. Set $\ell=\frac{k+1}{2}$ and consider $L_{1} \in \widetilde{\mathcal{L}}_{k+1, \ell}^{\nu, N}$ and $L_{2} \in \widehat{\mathcal{L}}_{k+1, \ell}^{\nu, N}$. Set $L=L_{1}+L_{2}$ and assume that for any $u \in \mathcal{E}$, $\underline{L}^{\ell}(u, \bar{u})$ is real valued. Then $\underline{L_{1}^{\ell}}(u, \bar{u})$ and $\underline{L_{2}^{\ell}}(u, \bar{u})$ are real valued.

Proof. Since $\underline{L}^{\ell}(u, \bar{u})$ is real valued, $\left\{\operatorname{Im} \underline{L}^{\ell}, G_{2}\right\}(u, \bar{u})=0$. As

$$
\left\{\underline{L}^{\ell}, G_{2}\right\}(u, \bar{u})=\left\{\underline{L}_{1}^{\ell}, G_{2}\right\}(u, \bar{u})
$$

by proposition 3.11, this yields $\left\{\operatorname{Im} \underline{L}_{1}{ }^{\ell}, G_{2}\right\}(u, \bar{u})=0$. Now, ii) of lemma 3.15 implies $\operatorname{Im} \underline{L_{1}^{\ell}}(u, \bar{u})=0$. Therefore, ${\underline{L_{1}}}^{\ell}(u, \bar{u})$ and $\underline{L_{2}}{ }^{\ell}(u, \bar{u})$ are real valued.

\subsection{Proof of theorem 2.6.}

We use a Birkhoff scheme to put the Hamiltonian system with the Hamiltonian $G$ of (2.19) in normal form. Having fixed some $r_{0} \geq 1$, the idea is to construct iteratively for $r=0, \ldots, r_{0}, \mathcal{U}_{r}$ a neighborhood of 0 in $H^{s}(M, \mathbb{C})$ for $s \gg 1$, a canonical transformation $\mathcal{T}_{r}$, defined on $\mathcal{U}_{r}$, an increasing sequence $\left(\nu_{r}\right)_{r=1, \ldots, r_{0}}$ of positive numbers, and functions $\mathcal{Z}^{(r)}, P^{(r)}, \mathcal{R}^{(r)}$ such that

$$
G^{(r)}:=G \circ \mathcal{T}_{r}=G_{2}+\mathcal{Z}^{(r)}+P^{(r)}+\mathcal{R}^{(r)} .
$$

Moreover, these functions will decompose as

$$
\begin{aligned}
\mathcal{Z}^{(r)} & =\sum_{j=1}^{r} \mathcal{Y}_{j} \\
P^{(r)} & =\sum_{j=r+1}^{r_{0}} Q_{j}^{(r)}
\end{aligned}
$$

where $\mathcal{Y}_{j}$ is in $\mathcal{H}_{j+2}^{s}\left(\nu_{j}\right)$ and Poisson commutes with $J_{n}$ for any $n, Q_{j}^{(r)}$ is in $\mathcal{H}_{j+2}^{s}\left(\nu_{r}\right)$, by convention $P^{\left(r_{0}\right)}=0$, and $\mathcal{R}^{(r)} \in C_{s}^{\infty}\left(\mathcal{U}_{r}, \mathbb{R}\right)$ has a zero of order $r_{0}+3$ at the origin. 
First remark that the Hamiltonian (2.19) has the form (3.31), (3.32), (3.33) with $r=0$ and $\mathcal{T}_{r}=I, P^{(0)}$ being the Taylor's polynomial of $\tilde{G}$ at degree $r_{0}$ (see lemma 3.5). We show now how to pass from $r$ to $r+1$ provided one is able to solve the homological equation below.

Lemma 3.17. Assume we are given $0<\nu_{r}$ and functions $\mathcal{Z}^{(r)}, P^{(r)}, \mathcal{R}^{(r)}$ satisfying the above conditions. Assume that there are $\nu_{r}^{\prime}>\nu_{r}$ and a function $F^{(r+1)}$ of $(u, \bar{u})$ with the properties that

$$
\begin{aligned}
F^{(r+1)} & \in \mathcal{H}_{r+3}^{s}\left(\nu_{r}^{\prime}\right) \\
\left\{F^{(r+1)}, G_{2}\right\} & \in \mathcal{H}_{r+3}^{s}\left(\nu_{r}^{\prime}\right) .
\end{aligned}
$$

Assume moreover one is able to choose $F^{(r+1)}$ with the further property that $\mathcal{Y}_{r+1}$ defined by

$$
\mathcal{Y}_{r+1}=\left\{F^{(r+1)}, G_{2}\right\}+Q_{r+1}^{(r)}
$$

Poisson commutes with $J_{n}$ for any $n$. Denote by $\Phi_{r+1}^{t}$ the flow generated by $X_{F^{(r+1)}}$. Then, there are $\nu_{r+1}>\nu_{r}^{\prime}$ and, for large enough $s$, a sufficiently small neighborhood $\mathcal{U}_{r+1}$ of the origin of $H^{s}(M, \mathbb{C})$, such that $G^{(r+1)}=G^{(r)} \circ \Phi_{r+1}^{1}$ has the same structure as $G^{(r)}$ but with $r$ replaced by $r+1$ and $\mathcal{U}_{r}$ replaced by $\mathcal{U}_{r+1}$.

Proof. If $\mathcal{U}_{r+1}$ is a sufficiently small neighborhood of the origin of $H^{s}(M, \mathbb{C})$, then $\Phi_{r+1}^{1}: \mathcal{U}_{r+1} \rightarrow \mathcal{U}_{r}$ is well defined. We decompose $G^{(r)} \circ \Phi_{r+1}^{1}$ as follows

$$
\begin{aligned}
G^{(r)} \circ \Phi_{r+1}^{1} & =G_{2}+\left\{F^{(r+1)}, G_{2}\right\}+Q_{r+1}^{(r)}+\mathcal{Z}^{(r)}+\mathcal{R}^{(r)} \circ \Phi_{r+1}^{1} \\
& +P^{(r)} \circ \Phi_{r+1}^{1}-Q_{r+1}^{(r)} \\
& +\mathcal{Z}^{(r)} \circ \Phi_{r+1}^{1}-\mathcal{Z}^{(r)} \\
& +G_{2} \circ \Phi_{r+1}^{1}-G_{2}-\left\{F^{(r+1)}, G_{2}\right\} .
\end{aligned}
$$

Using the fact that $\mathcal{Y}_{r+1}$ Poisson commutes with $J_{n}$ for any $n$ and belongs to $\mathcal{H}_{r+3}^{s}\left(\nu_{r}^{\prime}\right) \subset \mathcal{H}_{r+3}^{s}\left(\nu_{r+1}\right)$ by (3.35), we may define $\mathcal{Z}^{(r+1)}:=\mathcal{Z}^{(r)}+\mathcal{Y}_{r+1}$.

If $s$ is large enough, (3.34) implies that $F^{(r+1)} \in C_{s}^{\infty}\left(\mathcal{U}_{r}, \mathbb{R}\right)$, and we may apply lemma 2.5 with $F=F^{(r+1)}$ to $P^{(r)} \circ \Phi_{r+1}^{1}$ and $\mathcal{Z}^{(r)} \circ \Phi_{r+1}^{1}$. Using proposition 3.8 to write the iterated Poisson brackets of the right hand side of (2.30) in terms of multilinear forms, we thus see that (3.38), (3.39) may be decomposed in a sum of elements of $\mathcal{H}_{j+2}^{s}\left(\nu_{r+1}\right)$ for $s, \nu_{r+1}$ large enough and $j=r+2, \ldots, r_{0}$. Consequently these two terms will contribute to $P^{(r+1)}, \mathcal{R}^{(r+1)}$ in (3.31) written with $r$ replaced by $r+1$. In the same way, lemma 2.5 applied to $G_{2} \circ \Phi_{r+1}^{1}$ shows that, for large enough $r$ and $\nu_{r+1},(3.40)$ gives a contribution to $P^{(r+1)}+\mathcal{R}^{(r+1)}$ in (3.31) at step $r+1$. The conclusion follows.

Let us remark that the above lemma implies theorem 2.6. Actually, if we are able to apply lemma 3.17 up to step $r_{0}-1$, we get (3.31) with $r=r_{0}$, 
which is the conclusion of the theorem. Our remaining task is thus to solve the homological equation (3.36). This will be achieved in the following lemma.

Lemma 3.18. Let $2 \leq r \leq r_{0}+2, \nu \in \mathbb{R}_{+}^{*}$, and assume $m \in(0,+\infty)-\mathcal{N}$. For any $Q \in \mathcal{H}_{r+1}^{s}(\nu)$ there are $\nu^{\prime}>\nu, F \in \mathcal{H}_{r+1}^{s}\left(\nu^{\prime}\right)$ and $\mathcal{Y} \in \mathcal{H}_{r+1}^{s}(\nu)$, with $\mathcal{Y}$ which Poisson commutes with $J_{n}$ for any $n \geq 1$, such that

$$
\left\{F, G_{2}\right\}+Q=\mathcal{Y} \text {. }
$$

As a consequence one also has $\left\{F, G_{2}\right\} \in \mathcal{H}_{r+1}^{s}(\nu)$.

Proof. If $r+1$ is odd then we define $\mathcal{Y}=0$. As $Q$ is in $\mathcal{H}_{r+1}^{s}(\nu)$, it decomposes in the form

$$
Q=\sum_{\ell=0}^{r+1} \frac{L_{\ell}^{\ell}}{}
$$

where $L_{\ell}$ are multilinear forms in $\mathcal{L}_{r+1}^{\nu,+\infty}$. We remark that, since $r+1$ is odd, the $L_{\ell}$ are all non-resonant, i.e. $L_{\ell} \in \widetilde{\mathcal{L}}_{r+1, \ell}^{\nu,+\infty}$. Therefore by proposition 3.13 , we can define $F_{\ell} \in \widetilde{\mathcal{L}}_{r+1, \ell}^{\nu+\bar{\nu},+\infty}$ by

$$
F_{\ell}=-\mathrm{i} \Psi_{\ell}^{-1}\left(L_{\ell}\right)
$$

and in view of (3.21), the Hamiltonian function

$$
F=\sum_{\ell=0}^{r+1} \underline{F}_{\ell}^{\ell}
$$

satisfies the homological equation (3.41).

If $r+1$ is even, set $\tilde{L}_{\ell}=L_{\ell}$ if $\ell \neq \frac{r+1}{2}$. When $\ell=\frac{r+1}{2}$, write

$$
L_{\frac{r+1}{2}}=Y+\tilde{L}_{\frac{r+1}{2}} \in \widehat{\mathcal{L}}_{r+1, \frac{r+1}{2}}^{\nu,+\infty} \oplus \widetilde{\mathcal{L}}_{r+1, \frac{r+1}{2}}^{\nu,+\infty}
$$

using decomposition (3.19). Then if $\mathcal{Y}:=\underline{Y}^{\frac{r+1}{2}}$,

$$
Q-\mathcal{Y}=\sum_{\ell=0}^{r+1} \underline{\left(\tilde{L}_{\ell}\right)^{\ell}}
$$

and if we define $F$ by (3.44) with $F_{\ell}=-\mathrm{i} \psi_{\ell}^{-1}\left(\tilde{L}_{\ell}\right)$, we still obtain that equation (3.41) is satisfied.

It remains to show that $F$ is real valued. As $Q$ is real, using (3.42) yields for any $\ell \in\{0, \ldots, r+1\}$

$$
{\overline{L_{\ell}^{\ell}}}^{=}{\underline{L_{r+1-\ell}}}^{r+1-\ell}
$$


by homogeneity. If $r+1$ is even, (3.45) implies that $L_{\frac{r+1}{2}} \frac{r+1}{2}$ is real valued. Using lemma 3.16, we obtain that $\mathcal{Y}$ is real valued (remark that if $r+1$ is odd, $\mathcal{Y}=0$ is also real valued). Therefore, $\left\{F, G_{2}\right\}$ is real valued by (3.41). So $\left\{\operatorname{Im} F, G_{2}\right\}=0$ which implies by homogeneity that $\left\{\operatorname{Im} \underline{F_{\ell}^{\ell}}, G_{2}\right\}=0$ for any $\ell$. We may now use lemma 3.15 to obtain that $\operatorname{Im} \underline{F_{\ell}^{\ell}}=0$ for any $\ell \in\{0, \ldots, r+3\}$. Therefore, $F$ is real valued.

\subsection{Proof of theorem 2.2.}

Let $\mathcal{T}$ be the canonical transformation defined in theorem 2.6. Define on $\mathcal{U}=$ $\mathcal{T}(\mathcal{V})$ the function

$$
E(u, \bar{u}):=\sum_{n \geq 1} n^{2 s} J_{n} \circ \mathcal{T}^{-1}(u, \bar{u}) .
$$

We shall control $E(u, \bar{u})$ along long time intervals. To take into account the loss of derivatives coming from the linear part of the equation, we proceed by regularization. Fix $\sigma=s+1$ and take the Cauchy data such that $u_{0}=\epsilon\left(\Lambda_{m}^{-1 / 2} v_{1}+\right.$ $\left.\mathrm{i} \Lambda_{m}^{1 / 2} v_{0}\right) / \sqrt{2}$ is in $H^{\sigma}(M, \mathbb{C}) \cap \mathcal{U}$. Let $u(t) \equiv u(t,$.$) be the corresponding solution$ of $\dot{u}=X_{G}(u) \equiv \mathrm{i} \nabla_{\bar{u}} G(u, \bar{u})$. Since $X_{G}$ is semilinear and $H^{\sigma}$ is its domain, as far as $\|u(t)\|_{H^{s}}<\infty$ one has $u(t) \in H^{\sigma}$. Thus, as far as $u(t) \in \mathcal{U}$

$$
\frac{d E}{d t}=\partial E \cdot X_{G}=\{G, E\}
$$

which is well defined since $E \in C^{\infty}(\mathcal{U}, \mathbb{R})$, with $\mathcal{U} \subset H^{s}$ and $X_{G}(u) \in H^{s}$ for $u \in H^{\sigma}$. So we may write

$$
\{G, E\}(u, \bar{u})=\sum_{n \geq 1} n^{2 s}\left\{G, J_{n} \circ \mathcal{T}^{-1}\right\} .
$$

If we use (2.28), (2.33) and (2.34) we get then

$$
\begin{aligned}
\{G, E\} & =\sum_{n \geq 1} n^{2 s}\left\{G \circ \mathcal{T}, J_{n}\right\} \circ \mathcal{T}^{-1} \\
& =\sum_{n \geq 1} n^{2 s}\left\{G_{2}+\mathcal{Z}+\mathcal{R}, J_{n}\right\} \circ \mathcal{T}^{-1} \\
& =\left\{\mathcal{R} \circ \mathcal{T}^{-1}, E\right\} .
\end{aligned}
$$

Thus

$$
\frac{d E}{d t}=\left\{\mathcal{R} \circ \mathcal{T}^{-1}, E\right\}
$$

which, by taking an approximating sequence is seen to hold also for initial data which are not in $H^{\sigma}$, but only in $\mathcal{U}$. Using (2.35) one has

$$
\left|\frac{d E(t)}{d t}\right| \leq C\|u(t, \cdot)\|_{H^{s}}^{r+3} .
$$


Remark that by definition of $E(u, \bar{u})$ and because $\mathcal{T}(0)=0$, as long as $u$ stays in a small enough neighborhood of 0 , we have

$$
\frac{1}{2} E(u, \bar{u}) \leq\|u\|_{H^{s}}^{2} \leq 2 E(u, \bar{u})
$$

We deduce then by integration of (3.48) the estimate

$$
\|u(t, \cdot)\|_{H^{s}}^{2} \leq C^{\prime}\left(\left\|u_{0}\right\|_{H^{s}}^{2}+\left|\int_{0}^{t}\|u(\tau, \cdot)\|_{H^{s}}^{r+3} d \tau\right|\right)
$$

which holds true as long as $u$ remains in a small enough neighborhood of 0 . It is classical to deduce from this inequality that there are $C>0, c>0, \epsilon_{0}>0$ such that, if the Cauchy data $u_{0}$ is in the $H^{s}$ ball of center 0 and radius $\epsilon<\epsilon_{0}$, the solution exists over an interval of length at least $c \epsilon^{-r-1}$, and for any $t$ in that interval $\|u(t, \cdot)\|_{H^{s}} \leq C \epsilon$. This concludes the proof.

Remark 3.19. The proof of (2.37) is similar. As in (3.46) and (3.47), we see that

$$
\frac{d J_{n} \circ \mathcal{T}^{-1}(u, \bar{u})}{d t}=\left\{\mathcal{R} \circ \mathcal{T}^{-1}, J_{n}\right\}(u, \bar{u})
$$

which together with the bound $\|u(t, \cdot)\|_{H^{s}} \leq C_{1} \epsilon$ yields

$$
\left|J_{n} \circ \mathcal{T}^{-1}(u(t), \bar{u}(t))-J_{n} \circ \mathcal{T}^{-1}\left(u_{0}, \bar{u}_{0}\right)\right| \leq \frac{C \epsilon^{3}}{n^{2 s}}
$$

for times $|t| \leq \epsilon^{-r}$. Finally, using (3.51), (2.36) and the inequality

$$
\begin{aligned}
& \left|J_{n}(u(t), \bar{u}(t))-J_{n}\left(u_{0}, \bar{u}_{0}\right)\right| \leq\left|J_{n}(u(t), \bar{u}(t))-J_{n} \circ \mathcal{T}^{-1}(u(t), \bar{u}(t))\right| \\
& \quad+\left|J_{n} \circ \mathcal{T}^{-1}(u(t), \bar{u}(t))-J_{n} \circ \mathcal{T}^{-1}\left(u_{0}, \bar{u}_{0}\right)\right|+\left|J_{n}\left(u_{0}, \bar{u}_{0}\right)-J_{n} \circ \mathcal{T}^{-1}\left(u_{0}, \bar{u}_{0}\right)\right|
\end{aligned}
$$

implies (2.37).

\section{References}

[1] D. Bambusi and A. Giorgilli. Exponential stability of states close to resonance in infinite-dimensional Hamiltonian systems. J. Statist. Phys., 71(34):569-606, 1993.

[2] D. Bambusi and B. Grébert. Birkhoff normal form for pdes with tame modulus. Duke Math. J., To appear.

[3] D. Bambusi and N. N. Nekhoroshev. A property of exponential stability in the nonlinear wave equation close to main linear mode. Physica D, 122:73-104, 1998. 
[4] D. Bambusi. Birkhoff normal form for some nonlinear PDEs. Comm. Math. Physics, 234:253-283, 2003.

[5] J. Bourgain. Construction of approximative and almost-periodic solutions of perturbed linear Schrödinger and wave equations. Geometric and Functional Analysis, 6:201-230, 1996.

[6] J. Bourgain. On the growth in time of higher Sobolev norms of smooth solutions of Hamiltonian PDE. Internat. Math. Res. Notices, no. 6, 277304, 1996.

[7] J. Bourgain. Remarks on stability and diffusion in high-dimensional Hamiltonian systems and partial differential equations. Ergodic Theory Dynam. Systems, 24:1331-1357, 2004.

[8] P. R. Chernoff and J. E. Marsden. Properties of infinite dimensional Hamiltonian systems. Springer-Verlag, Berlin, 1974. Lecture Notes in Mathematics, Vol. 425.

[9] Y. Colin de Verdière. Sur le spectre des opérateurs elliptiques à bicaractéristiques toutes périodiques. Comment. Math. Helv., 54(3):508-522, 1979 .

[10] W. Craig and C. E. Wayne. Newton's method and periodic solutions of nonlinear wave equations. Comm. Pure Appl. Math., 46:1409-1498, 1993.

[11] J.-M. Delort. Temps d'existence pour l'équation de Klein-Gordon semilinéaire à données petites périodiques. Amer. J. Math., 120(3):663-689, 1998.

[12] J.-M. Delort. Existence globale et comportement asymptotique pour l'équation de Klein-Gordon quasi linéaire à données petites en dimension 1. Ann. Sci. École Norm. Sup. (4), 34(1):1-61, 2001.

[13] J.-M. Delort and J. Szeftel. Long-time existence for small data nonlinear Klein-Gordon equations on tori and spheres. Internat. Math. Res. Notices, 37:1897-1966, 2004.

[14] J.-M. Delort and J. Szeftel. Long-time existence for semi-linear KleinGordon equations with small Cauchy data on Zoll manifolds. Preprint, 2004.

[15] J.-M. Delort and J. Szeftel. Bounded almost global solutions for non Hamiltonian semi-linear Klein-Gordon equations with radial data on compact revolution hypersurfaces. Preprint, 2005. 
[16] J. J. Duistermaat and V. W. Guillemin. The spectrum of positive elliptic operators and periodic bicharacteristics. Invent. Math., 29(1):39-79, 1975.

[17] V. Guillemin. Lectures on spectral theory of elliptic operators. Duke Math. J., 44(3):485-517, 1977.

[18] S. Klainerman. The null condition and global existence to nonlinear wave equations. In Nonlinear systems of partial differential equations in applied mathematics, Part 1 (Santa Fe, N.M., 1984), volume 23 of Lectures in Appl. Math., pages 293-326. Amer. Math. Soc., Providence, RI, 1986.

[19] S. B. Kuksin. Nearly integrable infinite-dimensional Hamiltonian Systems. Springer-Verlag, Berlin, 1993.

[20] S. B. Kuksin and J. Pöschel. Invariant Cantor manifolds of quasi-periodic oscillations for a nonlinear Schrödinger equation. Ann. Math., 143:149-179, 1996.

[21] K. Moriyama, S. Tonegawa, and Y. Tsutsumi. Almost global existence of solutions for the quadratic semilinear Klein-Gordon equation in one space dimension. Funkcial. Ekvac., 40(2):313-333, 1997.

[22] J. Mujica. Complex analysis in Banach spaces, volume 120 of NorthHolland Mathematics Studies. North-Holland Publishing Co., Amsterdam, 1986. Holomorphic functions and domains of holomorphy in finite and infinite dimensions, Notas de Matemática [Mathematical Notes], 107.

[23] N. Nikolenko. The method of Poincaré normal form in problems of integrability of equations of evolution type. Russ. Math. Surveys, 41:63-114, 1986.

[24] T. Ozawa, K. Tsutaya, and Y. Tsutsumi. Global existence and asymptotic behavior of solutions for the Klein-Gordon equations with quadratic nonlinearity in two space dimensions. Math. Z., 222(3):341-362, 1996.

[25] J. Shatah. Normal forms and quadratic nonlinear Klein-Gordon equations. Comm. Pure Appl. Math., 38(5):685-696, 1985.

[26] A. Weinstein. Asymptotics of eigenvalue clusters for the Laplacian plus a potential. Duke Math. J., 44(4):883-892, 1977. 
D. Bambusi,

Università degli studi di Milano

Dipartimento di Matematica

Via Saldini 50

20133 Milano, Italy

J.-M. Delort

Laboratoire Analyse Géométrie et Applications, UMR CNRS 7539

Institut Galilée, Université Paris-Nord,

99, Avenue J.-B. Clément,

F-93430 Villetaneuse, France

B. Grébert

Laboratoire de Mathématiques Jean Leray, UMR CNRS 6629

Université de Nantes

2, rue de la Houssinière

F-44322 Nantes Cedex 03, France,

J. Szeftel

Department of Mathematics,

Princeton University,

Fine Hall, Washington Road

Princeton NJ 08544-1000 USA

and

Mathématiques Appliquées de Bordeaux, UMR CNRS 5466

Université Bordeaux 1

351 cours de la Libération

33405 Talence cedex, France 